\title{
Coating microbubbles with nanoparticles for medical imaging and drug delivery
}

\author{
"The major goal of bringing nanoparticles \\ and microbubbles together is to provide \\ multifunctionality."
}

First draft submitted: 13 October 2016; Accepted for publication: 26 October 2016; Published online: 23 November 2016

Keywords: microbubbles • nanoparticles • targeting delivery • ultrasound

In 1968, Gramiak and Shah discovered that small gaseous particles (i.e., microbubbles) between 1 and $10 \mu \mathrm{m}$ scattered ultrasound waves during an echocardiogram [1] and enhanced the contrast of the ultrasound image. Beyond ultrasound contrast enhancement, ultrasound-activated microbubbles enhance the transport of therapeutic agents by imposing a plethora of tunable mechanical effects (e.g., microstreaming, shear forces, shockwaves, membrane cavitation and jetting). Thus, ultrasound-enhanced strategies are employed across a broad range of biomedical applications including cancer and cardiovascular therapy, and diagnosis [2]. Considering their immense potential as theranostic agents, researchers have engineered microbubbles to improve circulation time, enable drug loading, increase stability and provide molecular targeting [3].

Early formulations for microbubbles utilized air as the gas core. They include Echovist, the first commercial contrast agent approved in Europe in 1991 and Albunex, the first microbubble-based contrast agent approved by the US FDA in 1994 [4]. However, air-filled microbubbles dissolve rapidly in the blood due to rapid surface tension driven dissolution or coalescence [5]. In order to prolong the life span of microbubbles, inert gases with high molecular weight, low solubility and diffusivity (e.g., perfluorocarbons and sulfur hexafluoride) could be used as the core [6]. Alternatively, the bubbles can be stabilized with a shell of lipids, polymers and proteins against the interface to reduce surface tension and prevent coalescence. The shell of the microbubble can play a role in clearance time and response to an ultrasound wave. Furthermore, it can act as a platform for the delivery of therapeutics [7].

Diverse hard shells including surfactants, lipid, proteins, polymers and a combination of them have been investigated. The shell material determines how easily a microbubble is taken up by the immune system and also affects the mechanical elasticity of the microbubble. More importantly, it brings new characteristics to the bubbles. For example, lipidcoated microbubbles are formed through the spontaneous self-assembly of phospholipids into a highly oriented and densely packed monolayer at the gas-liquid interface [8]. The lipid shell reduces the surface tension through lateral compression of monolayer plane and prevents the induction of Laplace overpressure on the curved interface that leads to dissolution of gas core and coalesces. Due to its weak van der Waals force between the lipid molecules, microbubbles can undergo oscillation in presence of acoustic signal. This allows the cargo, preloaded on the surface to be easily released from the microbubbles during inertial cavitation, making it a desirable drug carrier. Synthetic polymers are another choices [9]. The shell made of polymer is relatively rigid and does not oscillate actively. Therefore, during insonification, the polymeric shell fractures and encapsulated gas escapes. This phenomenon is not favored in

\section{Li Min Tay}

School of Chemical \& Biomedical Engineering, Nanyang Technological University, 62 Nanyang Drive, 637459, Singapore

and

Nanyang Institute of Technology in Health \& Medicine, Interdisciplinary Graduate School, Nanyang Technological University, Singapore

\section{Chenjie Xu}

Author for correspondence: School of Chemical \& Biomedical Engineering, Nanyang Technological University, 62 Nanyang Drive, 637459, Singapore

and

NTU-Northwestern Institute for Nanomedicine, Nanyang Technological University, 50 Nanyang Avenue, 639798, Singapore

CJXu@ntu.edu.sg 
acoustic imaging, but the fragmentation of the polymeric shell under the acoustic simulation attracts attention from the drug delivery field [10]. The fragmentation and accompanying jetting under high acoustic pressure have been shown to be efficient at propelling coated drugs into cells. The versatility of polymers also allows the incorporation of a wide variety of hydrophobic macromolecules within the polymeric matrix.

\section{Nanoparticle-coated microbubbles}

Recently, the use of nanoparticles (NPs) as the coating materials attracts lots of interest. The major goal of bringing NPs and microbubbles together is to provide multifunctionality. In addition, this complexion stabilizes the bubbles through modifying the interfacial tension and diffusivity of a gas bubble in liquid [11]. We group these efforts into two directions: multimodal contrast agents and theranostic platforms.

\section{NP-microbubble complexes as multimodal contrast agents}

Microbubbles as contrast agents for ultrasound imaging are already well established. However, it cannot be used to study problems of the lung and digestive tract that contain gas or calcified areas in the body (e.g., bones, plaques and hardened arteries). NPmicrobubble complexes are currently under development to leverage the capabilities of two or more imaging modalities.

\section{"These magnetic microbubbles offer sustainable release of drug-loaded microbubbles, yet are able to avoid the adverse cavitation effect."}

For instance, gold nanorods can be conjugated on the lipid shell of the microbubbles through the gold-thiol linkage under the presence of the (pyridyldithio)propionyl group [12]. This combination provides contrast enhancement for both photoacoustic and ultrasound imaging. Through a pilot in vivo imaging study with a photoacoustic imaging system, the researchers achieved $16.8 \pm 3.7 \mathrm{~dB}$ ultrasound and $5.7 \pm 1.6 \mathrm{~dB}$ photo-acoustic contrast enhancement from this complex. Ke et al. loaded quantum dots onto the microbubbles via the electrostatic layer-by-layer technique [13]. The acoustic and fluorescent information were integrated to produce a synthetic or hybrid image. On the one hand, this complex was able to respond to the medical ultrasound to act as contrast-enhanced agents. When exposed to high intensity ultrasound beams, the modified microbubbles were destroyed for the localized release of the loaded quantum dots at abnormal sites (e.g., tumors) for tissue or cell fluorescent imaging. Yang et al. embedded iron oxide NPs into the polymeric shell of nitrogen-filled microbubbles to enhance magnetic susceptibility [14].
Their in vivo MRI experiments over the rat liver showed that the magnetic NP-microbubble complex had longer contrast-enhancement duration in rat liver than plain microbubbles.

\section{NP-microbubble complexes as theranostic platforms}

Microbubbles have been well known as potential drug carriers. Drugs are commonly embedded in the polymeric or lipid shell of microbubbles. When insonified under specific ultrasound regimen, microbubbles undergo oscillations and induce acoustic cavitation, allowing an increase in cell membrane permeability in the close vicinity of the bubbles. This enables drugs to cross the membrane, thus penetrating into cells and ultimately inducing a therapeutic effect. On the same principle, microbubbles can also act on vessel permeability for promoting drug access to the extracellular space. For example, doxorubicin (DOX), a chemotherapeutic drug, could be placed in the superparamagnetic iron oxide NPs coated with poly(lactic-co-glycolic acid) (PLGA) shell of perfluorocarbon microbubbles [15]. This complex could enhance both ultrasound imaging and MRI in vivo. In the rabbit tumor metastasis model, treatment with these microbubbles could greatly limit the tumor progression [15]. Chemotherapeutical drug for glioblastoma multiforme, 1,3-bis(2-chloroethyl)-1-nitrosourea, could also be embedded in the lipid shell of perfluoropropane microbubbles to achieve local bloodbrain barrier opening and increase the penetration of therapeutic drugs into brain tumors [16].

There are a few issues about the above embedding strategy. First, the highly hydrophilic drugs such as nucleic acids would be difficult to embed into the shell. The addition of drugs to the shell can impact the bubble stability by increasing the aggregation and stiffness. In addition, the drug loading capacity is limited by the thin microbubble shell and gas core. Final, other drug carriers such as polymeric NPs can achieve sustained release after administration that allows a reduction in dosage frequency. To address these challenges, it is a natural choice to bring in drug-containing NPs into the microbubble system. Hydrophilic drugs can be easily encapsulated within NPs such as liposomes. NPs conjugated on the surface of microbubbles possess minimal impact over the physical properties of microbubbles. Furthermore, with drug-containing NPs being delivered through microbubbles, sustained release will be easily realized by NPs themselves [17,18].

The effort started as early as 2007, when Kheirolomoon et al. bound liposomes onto the microbubbles through the biotin-avidin affinity system [19]. Taking a fluorophore (NBD-cholesterol) as a model drug, they showed that microbubbles carrying liposomes could 
be spatially concentrated on a monolayer of cells at the focal point of ultrasound beam. Liposomes then fused with the cells and internalization of NBD occurred shortly after incubation. The biotin-avidin linkage is realizable, but it is not ideal for in vivo use because it can induce an immunogenic response. Thus maleimidethiol chemistry was used instead. Geers et al. prepared DOX liposome coated perfluorobutane microbubbles by maleimide conjugation [20]. Ultrasound-induced microbubble collapse clearly stimulated the release of DOX. Along the success of liposome delivery, polymeric NPs are gradually introduced into the microbubble system as well. Burke et al. coupled PLGA NPs to albumin microbubbles through carbodiimide chemistry [21]. Although no drug was encapsulated within NPs, this composite plus the ultrasound treatment enhanced the NP delivery to skeletal muscle 8- to 18 -fold over 'no ultrasound' controls. Wang and colleagues embedded curcumin-loaded albumin NPs in the lipid shell of microbubbles [17]. By doing so, there was an increase of $297 \%$ in drug loading efficiency if drugs were first loaded to NPs before being placed on the microbubbles.

Microbubbles are heavily explored in the delivery of drugs or drug-containing NPs as discussed above. However, this delivery relies on the collapse of microbubbles, which will produce shockwave/microjets that transport the therapeutic agents across the endothelial vasculature. The inertial cavitation of microbubbles can cause damage to the surrounding tissues of the target site. Furthermore, the sudden burst release of drugloaded NPs may cause toxicity. Recently, our group discovered that the vibration of gas bubbles upon the ultrasound treatment could provide momentum for the drug-containing NPs to detach from the bubbles and penetrate into the tissue [22]. Specifically, microbubbles that were subjected to sufficient high resonant ultrasound, underwent high-order shape oscillation modes. Then NPs began to shed off a few hundred micrometers away from the microbubbles. When majority of NPs were detached from microbubbles, the bubbles shrank and disappeared. These magnetic microbubbles offer sustainable release of drug-loaded microbubbles, yet are able to avoid the adverse cavitation effect [22]. As shown in our preliminary study, DOX-containing PLGA NPs were able to penetrate as deep as $200 \mu \mathrm{m}$ into the tumor tissue when it was released from microbubbles in vivo under the ultrasound stimulation.

\section{Challenges}

The integration of NPs and microbubbles is a new and emerging direction. This complexation brings potential to targeted multimodal imaging and theranostics. However, to further translate this platform to clinics, there are a few challenges to be addressed.

\section{Composition \& stability of NP-microbubble complex}

Any formulation to be used in human has to be precisely controlled about their composition. Currently, NPs are mainly loaded onto the microbubbles either through conjugation/ligand recognition, such as biotin-avidin interaction or through the physical absorption like electrostatic interaction. When NPs are loaded through any of these methods, the receptors on the bubble surface should be preciously controlled. Unfortunately, there has not been any report to address this question [18,23]. In addition, due to the direct contact with blood/body fluid, NPs on the surface of microbubbles might detach from the bubbles. This would present challenges to predict the biodistribution of NPs. One temporal solution is to co-deliver NPs and microbubbles that are well characterized separately. For example, Chertok et al. co-delivered heparin-engrafted DNA liposomes with microbubbles into tumor-bearing mice intravenously. The tumordirected ultrasound-targeted microbubble destruction facilitates these DNA nanoplexes and gene transfer to the tumor site [24].

\section{"This complexation brings potential to targeted multimodal imaging and theranostics."}

\section{Standardization of the administration \&} manipulation of NP-microbubble complex

NP-microbubble complexes usually have a size 4-10 $\mu \mathrm{m}$, slightly smaller than red blood cells. Both systematic injection and local injection at disease sites are seen in literature for administering them in animal models. However, the specific administration method for different diseases is not clearly defined. Furthermore, ultrasound is a key component in the application of NP-microbubble complex, especially for the controlled drug delivery. Its frequency, intensity and position to the microbubbles are all critical for the release of NPs from NP-microbubble complex. Unfortunately, few reports placed emphasis on this issue.

\section{Conclusion}

NP-coated microbubbles are a platform for the realization of multimodal imaging and targeted theranostics. Relying on the noninvasiveness of ultrasound imaging and stimulation, this platform aims to improve the resolution, precision and accuracy in the medical imaging and the targeted yet controlled drug delivery. With the efforts to address the existing challenges, this trend will push the microbubble field into a new era.

\section{Acknowledgements}

We thank JJ Kwan for reading this paper and suggesting many improvements. 
Financial \& competing interests disclosure

The work was supported by Ministry of Education Tier 1 Academic Research Fund (RG131/15) and NTU-Northwestern Institute for Nanomedicine (M4081502.F40). The authors have no other relevant affiliations or financial involvement with any

\section{References}

1 Karamanou M, Papaioannou TG, Stefanadis C, Androutsos G. Genesis of ultrasonic microbubbles: a quick historical overview. Curr. Pharm. Des. 18(15), 2115-2117 (2012).

2 Lindner JR. Microbubbles in medical imaging: current applications and future directions. Nat. Rev. Drug Discov. 3(6), 527-532 (2004).

3 Martin KH, Dayton PA. Current status and prospects for microbubbles in ultrasound theranostics.. Wiley Interdiscip. Rev. Nanomed. Nanobiotechnol. 5(4), 329-345 (2013).

4 Lindner JR. Microbubbles in medical imaging: current applications and future directions. Nat. Rev. Drug Discov. 3(6), 527-533 (2004).

5 Kwan JJ, Borden MA. Microbubble dissolution in a multigas environment. Langmuir 26(9), 6542-6548 (2010).

6 Anselmo AC, Mitragotri S. Nanoparticles in the clinic. Bioeng. Transl. Med. 1(1), 10-29 (2016).

7 Lee M, Lee EY, Lee D, Park BJ. Stabilization and fabrication of microbubbles: applications for medical purposes and functional materials. Soft Matter 11(11), 2067-2079 (2015)

8 Sirsi S, Borden M. Microbubble compositions, properties and biomedical applications. Bubble Sci. Eng. Technol. 1(1-2), 3-17 (2009).

9 Xiong X, Zhao F, Shi M, Yang H, Liu Y. Polymeric microbubbles for ultrasonic molecular imaging and targeted therapeutics. J. Biomater. Sci. Polym. Ed. 22(4-6), 417-428 (2011).

10 Hernot S, Klibanov AL. Microbubbles in ultrasoundtriggered drug and gene delivery. Adv. Drug Delivery Rev. 60(10), 1153-1166 (2008).

11 Azmin M, Mohamedi G, Edirisinghe M, Stride EP. Dissolution of coated microbubbles: the effect of nanoparticles and surfactant concentration. Mater. Sci. and Eng. C 32(8), 2654-2658 (2012).

12 Dixon AJ, Hu S, Klibanov AL, Hossack JA. Oscillatory dynamics and in vivo photoacoustic imaging performance of plasmonic nanoparticle-coated microbubbles. Small 11(25), 3066-3077 (2015).

13 Ke H, Xing Z, Zhao B et al. Quantum-dot-modified microbubbles with bi-mode imaging capabilities. Nanotechnology 20(42), 425105 (2009). organization or entity with a financial interest in or financial conflict with the subject matter or materials discussed in the manuscript apart from those disclosed.

No writing assistance was utilized in the production of this manuscript.

14 Yang F, Li Y, Chen Z, Zhang Y, Wu J, Gu N. Superparamagnetic iron oxide nanoparticle-embedded encapsulated microbubbles as dual contrast agents of magnetic resonance and ultrasound imaging. Biomaterials 30(23-24), 3882-3890 (2009).

15 Niu C, Wang Z, Lu G et al. Doxorubicin loaded superparamagnetic PLGA-iron oxide multifunctional microbubbles for dual-mode US/MR imaging and therapy of metastasis in lymph nodes. Biomaterials 34(9), 2307-2317 (2013).

16 Fan C-H, Ting C-Y, Chang Y-C, Wei K-C, Liu H-L, Yeh C-K. Drug-loaded bubbles with matched focused ultrasound excitation for concurrent blood-brain barrier opening and brain-tumor drug delivery. Acta Biomater. 15 89-101 (2015).

17 Wang J, Li P, Tian R et al. A novel microbubble capable of ultrasound-triggered release of drug-loaded nanoparticles. J. Biomed. Nanotechnol. 12(3), 516-524 (2016).

18 Mullin LB, Phillips LC, Dayton PA. Nanoparticle delivery enhancement with acoustically activated microbubbles. IEEE Trans. Ultrason. Ferroelect. Freq. Control 60(1), 65-77 (2013).

19 Kheirolomoom A, Dayton PA, Lum AFH et al. Acoustically-active microbubbles conjugated to liposomes: characterization of a proposed drug delivery vehicle. J. Control. Release 118(3), 275-284 (2007).

20 Geers B, Lentacker I, Sanders NN, Demeester J, Meairs S, De Smedt SC. Self-assembled liposome-loaded microbubbles: the missing link for safe and efficient ultrasound triggered drug-delivery. J. Control. Release 152(2), 249-256 (2011).

21 Burke CW, Hsiang Y-HJ, Alexander E, Kilbanov AL, Price RJ. Covalently linking poly(lactic-co-glycolic acid) nanoparticles to microbubbles before intravenous injection improves their ultrasound-targeted delivery to skeletal muscle. Small 7(9), 1227-1235 (2011).

22 Gao Y, Chan CU, Gu Q et al. Controlled nanoparticle release from stable magnetic microbubble oscillations. NPG Asia Mater. 8, e260 (2016).

23 Owen J, Pankhurst Q, Stride E. Magnetic targeting and ultrasound mediated drug delivery: benefits, limitations and combination. Int. J. Hyperthermia 28(4), 362-373 (2012).

24 Chertok B, Langer R, Anderson DG. Spatial control of gene expression by nanocarriers using heparin masking and ultrasound-targeted microbubble destruction. ACS Nano 10(8), 7267-7278 (2016). 